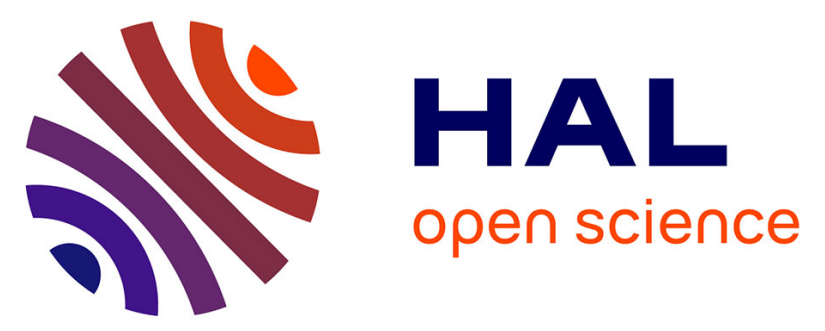

\title{
Binder and interphase microstructure in a composite material characterized by scanning electron microscopy and NMR spin diffusion experiments
}

Silène Parisse, Jean-fabien Petit, Alexandre Forzy, Alexandre Lecardeur, Sandrine Beaugrand, Pascal Palmas

\section{To cite this version:}

Silène Parisse, Jean-fabien Petit, Alexandre Forzy, Alexandre Lecardeur, Sandrine Beaugrand, et al. Binder and interphase microstructure in a composite material characterized by scanning electron microscopy and NMR spin diffusion experiments. Macromolecular Chemistry and Physics, 2019, 221 (2), pp.1900310. 10.1002/macp.201900310 . hal-03417321

\author{
HAL Id: hal-03417321 \\ https://hal.science/hal-03417321
}

Submitted on 30 Nov 2021

HAL is a multi-disciplinary open access archive for the deposit and dissemination of scientific research documents, whether they are published or not. The documents may come from teaching and research institutions in France or abroad, or from public or private research centers.
L'archive ouverte pluridisciplinaire HAL, est destinée au dépôt et à la diffusion de documents scientifiques de niveau recherche, publiés ou non, émanant des établissements d'enseignement et de recherche français ou étrangers, des laboratoires publics ou privés.

\section{(ㅇ)(1) $\$$}

Distributed under a Creative Commons Attribution - NonCommerciall 4.0 International 


\title{
Binder and Interphase Microstructure in a Composite Material Characterized by Scanning Electron Microscopy and NMR Spin Diffusion Experiments
}

\author{
Silène Parisse, Jean-Fabien Petit, Alexandre Forzy, Alexandre Lecardeur, Sandrine \\ Beaugrand, and Pascal Palmas
}

\begin{abstract}
${ }^{1} \mathrm{H}$ NMR spin diffusion is shown to advantageously complement scanning electron microscopy (SEM) observations for the characterization of polymer structuring in composite materials. It is here demonstrated on a material containing a few percent of polymer binder and a crystalline organic/inorganic mixture as reinforcement. In SEM observations, polymer accumulations are seen. However, the polymer is also expected to fill small porosities and thin layers at the interface of particles to ensure the cohesion and the mechanical properties of the assembly. In most cases, this polymer structuring is invisible considering the resolution and contrast achieved by SEM on such material. It is thus investigated taking advantage of the two-step decay of nuclear magnetic resonance spin diffusion curves. Average thickness values of 30 and $188 \mathrm{~nm}$ are estimated for the interphase and the overall polymer, respectively. Such structural information improves the knowledge of structure/property relationships and provides better understanding of material properties and making processes.
\end{abstract}

of the reinforcement particles and the interphase between the polymer and the reinforcement. The adhesion at the interface is of prime importance regarding mechanical performance, and is related to the ability to produce a fine mixture of both species during the manufacturing step. This operation is even more difficult whenever the amount of polymer is considerably reduced. The final result thus depends on the surface properties of the particles and the mutual affinity between the constituents.

The characterization of the location of the binder within these composite materials has been addressed in our laboratory by optical microscopy and scanning electron microscopy (SEM) and also using Raman spectroscopy and energy dispersion spectroscopy (EDS). The complex character of the structuring was demonstrated showing that the polymer is mainly located in large regions disseminated throughout the sample. However, the presence of polymer in other places, especially at the interface between grains, was not clear considering the resolution and contrast that could be achieved with our materials. Despite these limitations, such spatially resolved information is very important as it gives a direct picture of the binder and its structuring. To evaluate the mixture between the polymer and the reinforcement for a specific sample, it is more convenient to extract from the images some parameters that give structural information averaged out over the sample. A quantitative image analysis is thus required to reduce the number of data points to a limited number of reliable parameters allowing a correct description of polymer morphology. Work in this field is currently ongoing in our laboratory, together with improvements in experimental SEM spatial resolution.

Another possibility to obtain average structural information is the measurement of overall parameters that depend on polymer structuring. Solid-state NMR spectroscopy is widely used for molecular structure investigations and is sensitive to the close molecular environment. A supramolecular organization over longer distances in solids can also be probed by taking advantage of the spin diffusion process. Such an experiment was first suggested by Goldman and Shen ${ }^{[1]}$ (GS) in 1966 who investigated spin-spin relaxation of lanthanum trifluoride.
S. Parisse, Dr. J.-F. Petit, A. Forzy, A. Lecardeur, Dr. S. Beaugrand,

Dr. P. Palmas

CEA DAM le Ripault

F-37260 Monts, France

E-mail: pascal.palmas@cea.fr 
Over the next years, several studies have shown the relevance of using the GS approach to characterize domain sizes at the nano-scale in amorphous polymers, semi-crystalline polymers, ${ }^{[2-5]}$ and copolymers. ${ }^{[6,7]}$ Moreover, it has been used to investigate the structure and dynamics of different phases ${ }^{[6]}$ and interphases ${ }^{[8]}$ in polymer blends. Works have also been reported on biopolymers ${ }^{[4]}$ and hybrid chitosan-polymer nanoparticles for clinical application. ${ }^{[9]}$ Conversely, only a few papers dealing with the characterization of nanocomposite materials have been reported. ${ }^{[10-12]}$ The efficiency of the method has been recognized for the characterization of domains with length scales in the range of several nanometers to a few tens of nanometers. It is admitted that the material structuring effect vanishes around $200 \mathrm{~nm}$, depending on the system characteristics. There thus exists a real complementarity between observations with microscopic techniques that give spatially resolved information with limited resolution, and NMR spin diffusion approach that provides overall structural information averaged over a sample with a higher resolution.

In this paper we have investigated the potential of the GS experiment to characterize one of our materials that contains only a few percent of polymer binder. This soft component is diluted within the reinforcement composed of rigid crystalline compounds. To the best of our knowledge, a spin diffusion study applied to such a material has yet to be reported. Published works generally concern cases where fractions of soft and rigid domains are in the same range, while the few studied composite materials are made of polymers only.

\section{Theoretical Background}

\subsection{Spin Diffusion NMR}

Spin diffusion experiments are based on the existence of homonuclear dipolar couplings and differences in molecular mobility between two spatially separated regions within one material. These differences in mobility allow the selection of either the mobile or the rigid region, which can be performed using the Goldman-Shen sequence, ${ }^{[1]}$ a dipolar filter as proposed by Spiess et al. ${ }^{[13-16]}$ or Demco et al., ${ }^{[17-19]}$ or using a double quantum ${ }^{[18,20-22]}$ filter. In the case of Golman-Shen or dipolar filter, the magnetization of the mobile component is preserved while the magnetization of the rigid component is suppressed. For a double quantum filter, the inverse phenomenon is observed: coherences with dipolar couplings are created, which allow the preservation of the rigid component's magnetization. Another kind of selection utilizes chemical shift differences that can be resolved with cross-polarization magic-angle spinning (CP/MAS) or combined rotation and multiple pulse sequence (CRAMPS) detection techniques. It can be carried out in one dimension ${ }^{[23]}$ or two dimensions as in the nuclear overhauser effect spectroscopy (NOESY). ${ }^{[24]}$ Very often, ${ }^{1} \mathrm{H}$ detection is performed, but it is sometimes more convenient to detect ${ }^{13} \mathrm{C}$ with the CP procedure. ${ }^{[3]}$ This procedure improves selectivity for detection with the drawback of reducing the spin diffusion rate. ${ }^{[24]}$ Diffusion can also be monitored in a two dimensional homonuclear ${ }^{[23]}$ or heteronuclear ${ }^{[6]}$ experiment.

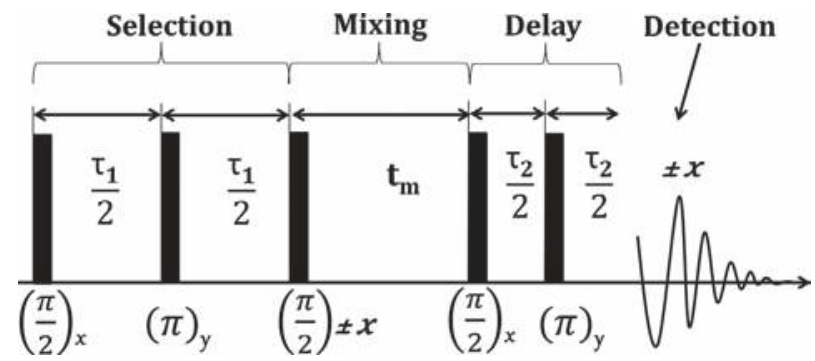

Figure 1. Sequence based on the Goldman-Shen sequence: selection, mixing, and detection. The phase cycling between the phase of the second $\pi / 2$-pulse and the acquisition is added to reduce the $T_{1}$ effect, and two $\pi$-pulses are added to refocus the signal.

In our case, ${ }^{1} \mathrm{H}$ detection is mandatory as there is not enough polymer within our materials to allow ${ }^{13} \mathrm{C}$ detection.

The Goldman-Shen sequence is the simplest scheme to demonstrate the effect of spin diffusion by selecting the mobile region. Improvements have already been suggested either to reduce the $T_{1}$-effect ${ }^{[6]}$ or to increase the selection efficiency. A variant of the original pulse sequence is suggested by inserting two $180^{\circ}$ pulses: one during the selection, the other before the detection, as shown in Figure 1.

These two pulses aim at refocusing the polymer magnetization while removing the hard short- $T_{2}$ component. The delay of the spin echo represents a tunable filter for selection of domains with different mobility. Moreover, since large domains are expected in our material, long distances have to be probed by spin diffusion. This has two consequences: i) the MAS procedure is not used even for ${ }^{1} \mathrm{H}$ as it would significantly reduce the diffusion coefficients; ii) the analysis cannot be limited to the initial part of the diffusion curve as knowledge of the diffusion behavior at long mixing times is necessary. Hence, the $T_{1}$ relaxation effect has to be removed from the observed evolution. This is achieved thanks to a phase cycling of the second $90^{\circ}$ pulse and the phase of the receptor ${ }^{[6,25]}$ at the same time. However, the relaxation effect is not completely canceled and there is still an attenuation of the form $R(t)=\exp \frac{-t}{T_{1}} \cdot[24,25]$ Finally, dividing the experimental attenuation by the correction function $R(t)$ should give a pure diffusion curve.

When limiting the analysis to the beginning of the curve, this correction is weak and can be neglected, but it is crucial for long mixing times due to the exponential dependency. Nevertheless, the longer the mixing time, the higher the impact of a small $T_{1}$ inaccuracy on the final curve shape. Hence, instead of measuring $T_{1}$ with an independent specific sequence, the correction curve is determined by recording a reference experiment with the same sequence but zero $\tau 1$-delays. In such conditions, no selection is performed, so that both magnetizations of rigid and mobile parts are preserved at the beginning of the mixing period. Therefore, the observed evolution as a function of $t_{\mathrm{m}}$ only depends on $T_{1}$ relaxation. An individual reference is recorded after each experiment, to avoid any deviation of experimental settings. The $T_{1}$-corrected diffusion curve is finally calculated according to the ratio:

$I(t)_{\mathrm{corr}}=\frac{I(t)_{\tau}}{I(t)_{\tau=o \mu \mathrm{sec}}}$ 
Where $I(t)_{\tau}$ and $I(t)_{\tau=0 \text { usec }}$ are the diffusion curve and reference, respectively. $I(t)_{\text {corr }}$ is then normalized according to the intensity at $t_{\mathrm{m}}=0$ obtained by a linear extrapolation of the first points.

\subsection{Treatment of Diffusion Curves}

Domain size information is extracted from diffusion curves within the framework of Fick's second law formalism. Numerical approaches ${ }^{[25]}$ and analytical solutions have been reported to deal with systems containing up to three morphological domains, that is, two phases A and B separated by an interphase region $\mathrm{I}^{[19]}$. In some cases, domain sizes have been determined by fitting the whole curve ${ }^{[3,8,19]}$ but generally, the analysis is restricted to the behavior at short mixing times. In that case, the initial part of the curve is assumed as linear, and its intercept with the equilibrium plateau value reached for long mixing times gives $\sqrt{t_{m}^{s}}$. Mathematical relationships ${ }^{[13]}$ can be found in the literature for a two-phase system, where this term is related to the domain size. We use the formulation given by Clauss, Schmidt-Rohr, and Spiess in $1993^{[26]}$ that yields the domain size of the dispersed component.

$d_{\mathrm{dis}}=\left(\frac{\rho_{\mathrm{HA}} \varphi_{\mathrm{A}}+\rho_{\mathrm{HB}} \varphi_{B}}{\varphi_{A} \varphi_{B}}\right) \frac{4 \varepsilon \varphi_{\mathrm{dis}}}{\sqrt{\pi}} \frac{\sqrt{\left(D_{A} D_{B}\right)}}{\sqrt{D_{A}} \rho_{\mathrm{HA}}+\sqrt{D_{B}} \rho_{\mathrm{HB}}} \sqrt{t_{m}^{s}}$

Reaching equilibrium is not always possible as the maximum $t_{\mathrm{m}}$ value is limited by the relaxation time of the system, generally less than $1 \mathrm{~s}$ in polymers. It is thus convenient to use $\sqrt{t_{m}^{s, 0}}$, the intercept of the first slope with the $x$ axis, using the equivalence between the ratio $\sqrt{t_{m}^{s}} / \sqrt{t_{m}^{s, 0}}$ and the volume fraction of the sink phase $\phi \operatorname{sink}^{[13]}$ In our experiments, the sink phase is the dispersed rigid component. This then gives

$d_{r}=\left(\frac{\rho_{H r} \varphi_{r}+\rho_{H m} \varphi_{m}}{\varphi_{m}}\right) \frac{4 \varepsilon \varphi_{r}}{\sqrt{\pi}} \frac{\sqrt{\left(D_{r} D_{m}\right)}}{\sqrt{D_{r}} \rho_{H r}+\sqrt{D_{m}} \rho_{H m}} \sqrt{t_{m}^{s, 0}}$

with i) $D_{\mathrm{m}, \mathrm{r}}$ and $\rho_{\mathrm{Hm}, \mathrm{r}}$ the diffusion coefficient and proton density of the mobile and rigid components, $\rho_{H=\frac{N_{P} \rho}{M}}$ with $N_{\mathrm{P}}$ the number

Table 1. Parameters obtained for the polymer and for the composite. of hydrogen atoms per molecule, $M$ the molar mass, and $\rho$ the density; ii) $\phi_{\mathrm{m}}$ the proton fraction of the mobile component deduced from the final value of the selected fraction curve (Figures 6b and $7 \mathrm{~d}$ ); the rigid fraction is $\phi_{\mathrm{r}}=1-\phi_{\mathrm{m}}$; iii) $\varepsilon$ the dimensionality of the system, that is, the number of directions relevant for the spin diffusion process: $\varepsilon=1,2$, or 3 , respectively for lamellar, cylindrical, and spherical (or cubic) systems. These are limit cases, and real situations can be intermediate or contain a mixture of them.

Diffusion coefficients $D_{\mathrm{m}}$ and $D_{\mathrm{r}}$ can be estimated from ${ }^{1} \mathrm{H}$ NMR parameters as suggested by Demco et al. ${ }^{[17]}$

$D=\frac{1}{6}\left\langle r^{2}\right\rangle \sqrt{\alpha \Delta v_{\frac{1}{2}}}$

In our case, the full-width at half-height $\Delta v_{1 / 2}$ is not easy to determine, as the lines of the spectrum are convoluted. To overcome this problem, we measured $T_{2}$ relaxation times from a Hahn echo experiment and used the following equation instead which is valid for a Lorentzian line shape:

$D=\frac{1}{6}\left\langle r^{2}\right\rangle \sqrt{\frac{\alpha}{\pi T_{2}}}$

with $\alpha$ the "cutoff" parameter, determined from the spectrum as the linewidth beyond which each peak intensity is considered zero (i.e., below the noise level). For the narrower lines \#1 and \#2 (see Table 1), we obtained $\alpha=80 \mathrm{kHz}$ from the Hahn echo filtered spectrum. For the wider line, a value of $\alpha=200 \mathrm{kHz}$ was determined directly on the standard single pulse spectrum.

\section{Results}

\subsection{SEM Experiments}

The main drawback of the low-vacuum approach is, in addition to an inherent loss of sensitivity, the need to use a higher accelerating voltage. This increases the interaction volume and thus reduces the accessible resolution. Even if the pixel dimension can be reduced to a few nanometers, the measured intensity results from the average response of the whole interaction

\begin{tabular}{|c|c|c|c|c|c|c|}
\hline & $\begin{array}{c}\text { Frequencya) } \\
{[\mathrm{Hz}]}\end{array}$ & $\begin{array}{c}\Delta v_{1 / 2^{a}} \\
{[\mathrm{~Hz}]}\end{array}$ & $\begin{array}{c}\text { Area }^{a)} \\
{[\%]}\end{array}$ & $\begin{array}{l}T_{2}^{\mathrm{b})} \\
{[\mu \mathrm{s}]}\end{array}$ & $\begin{array}{c}\text { Corrected areac) } \\
{[\%]}\end{array}$ & $\begin{array}{c}\text { Diffusion coefficient }{ }^{\mathrm{d})} \\
{\left[\mathrm{nm}^{2} \mathrm{~ms}^{-1}\right]}\end{array}$ \\
\hline \multicolumn{7}{|l|}{ Polymer } \\
\hline$\# 1$ & 6075 & 1237 & 27 & 260 & 26 & 0.08 \\
\hline$\# 2$ & 4613 & 1542 & 56 & 260 & 55 & 0.08 \\
\hline \#3 & 4391 & 18239 & 17 & 38 & 19 & 0.25 \\
\hline \multicolumn{7}{|l|}{ Composite } \\
\hline$\# 1$ & 6075 & 2050 & 19 & 383 & 17 & 0.06 \\
\hline$\# 2$ & 4613 & 2469 & 23 & 383 & 20 & 0.06 \\
\hline$\# 3$ & 4256 & 49844 & 58 & $80 \& 14^{\mathrm{e})}$ & 59 & 0.22 and $0.53^{\mathrm{e})}$ \\
\hline Reinforcement & - & - & - & - & 4 & - \\
\hline
\end{tabular}

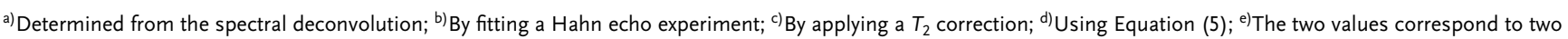
unresolved components that contribute to the broad line and are respectively attributed to the small domains and the interphase. 


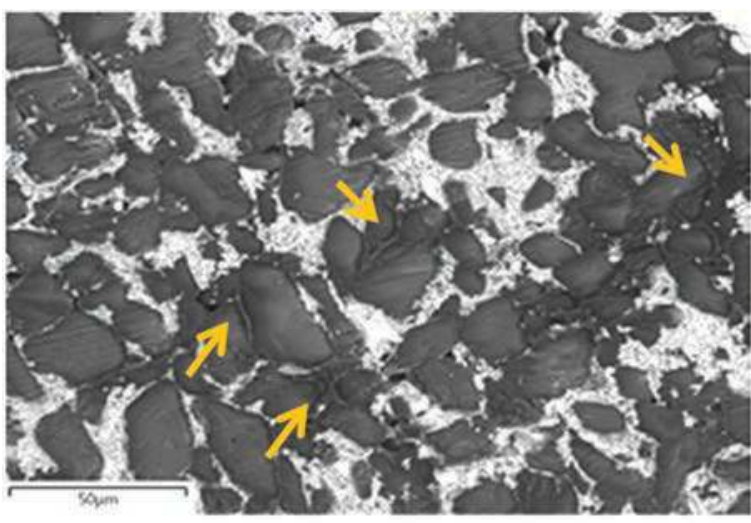

\section{K series}

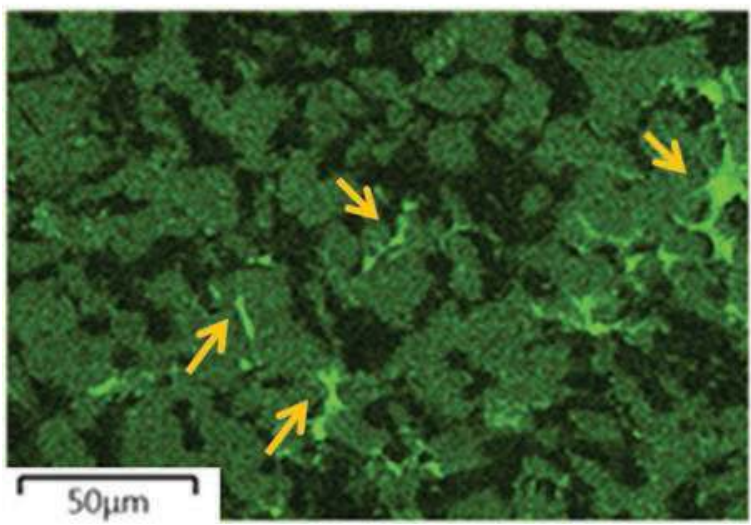

Figure 2. SEM backscattered electron image (top) and EDS-carbon cartography (bottom) of the composite material. The arrows show areas of polymer binder which appear darker in SEM and brighter in the EDScarbon cartography.

volume, limiting the discrimination between the polymer and the reinforcement. The final resolution cannot be known exactly but probably does not exceed a few hundred nanometers.

An SEM image of a representative region of the sample is displayed in Figure 2. A heterogeneous microstructure is revealed with dark melamine particles of different sizes separated by more or less extended bright areas of barium sulfate clusters. The bright response of this mineral component strongly reduces the dynamic range of grey levels that are available for the organic fraction of the sample. This effect renders difficult the distinction between melamine particles and polymer binder, both organic components. Nevertheless, darker areas are seen in several places of the SEM image. These areas give a more intense $\mathrm{X}$-ray response in the corresponding EDS map recorded for the carbon series. This is consistent with the presence of polymer binder in these places as this compound contains more carbon than melamine. The clear difference in the carbon signal intensity means that the polymer depth under these areas is at least a few hundred nanometers, given that the interaction depth with X-rays is about $1 \mu \mathrm{m}$ in soft materials. It is worth noticing that CBS and EDS detectors do not yield the same spatial information, mainly because backscattered electrons and X-rays interaction volumes are not identical. Small differences in geometrical details can thus be observed between both techniques.
From these observations, the polymer distribution is seen to be non-uniform. Local accumulations of polymer are observed in some places forming lamellar-like clusters a few microns wide and long between melamine particles. Some little contrast variations are also seen very locally within barium sulfate particles. However, the presence of polymer in these places is less clear since it cannot be confirmed by EDS. Surprisingly, the images contain large areas where the polymer is apparently missing. This is not consistent with the observed cohesion and mechanical properties of the material and the expected polymer content of a few percent. This non-detection probably means that spatial domains occupied by the polymer binder are too small to produce a visible response in both SEM and EDS experiments considering the resolution and contrast that can be achieved with such materials.

Information given by these observation techniques is primordial as they give a visual and direct representation of the structuring of the material at the sub-micrometer scale. However, it must be kept in mind that images are obtained from a clean surface with interaction volumes that are larger than geometrical details with dimensions of a pixel. Consequently, the real dimensions of polymer domains are hardly accessible and those with dimensions less than a few dozen nanometers are simply undetectable. Finally, extracting an average polymer thickness that makes sense would require a dramatic improvement of the accessible resolution and the analysis of a field of view large enough to be representative. Moreover, the assumption would have to be made that information given by a sufficiently large surface is representative of the whole sample volume.

Spin diffusion solid state NMR is a possibility to go further, since it gives information averaged over the whole sample and is sensitive to polymer structuring at the nanometer scale. It would thus unravel the presence of possible polymer domains smaller than those visible with SEM, and enrich our knowledge about the microstructure of the material.

\subsection{D NMR Spectra}

The neat polymer is studied first, to distinguish structural effects present within the polymer itself from effects due to an interaction with the reinforcement.

Figure 3 displays the ${ }^{1} \mathrm{H}$ spectra of the neat polymer and the composite recorded with a short recycle delay of $5 \mathrm{~s}$. The spectrum of neat polymer is dominated by two rather narrow lines separated by $\approx 3.4 \mathrm{ppm}$ and a small contribution of a broader component. The two narrow lines on the one hand represent protons that belong to aliphatic saturated and unsaturated carbons of the NBR moiety. The broad line on the other hand contains a major contribution from EPON that represents the most rigid part of the polymer. The spectrum of the composite was also recorded after a long delay and shown for comparison. In this case, the pattern is dominated by a broadened characteristic Pake doublet due to the contribution of the reinforcement.

A deconvolution procedure was applied to these spectra using DMFit software. A first deconvolution was obtained on a Hahn echo spectrum of the binder, to remove the broad component (not shown). Then, the whole spectrum was deconvoluted with fixed frequencies for the sharp lines, to get the parameters 

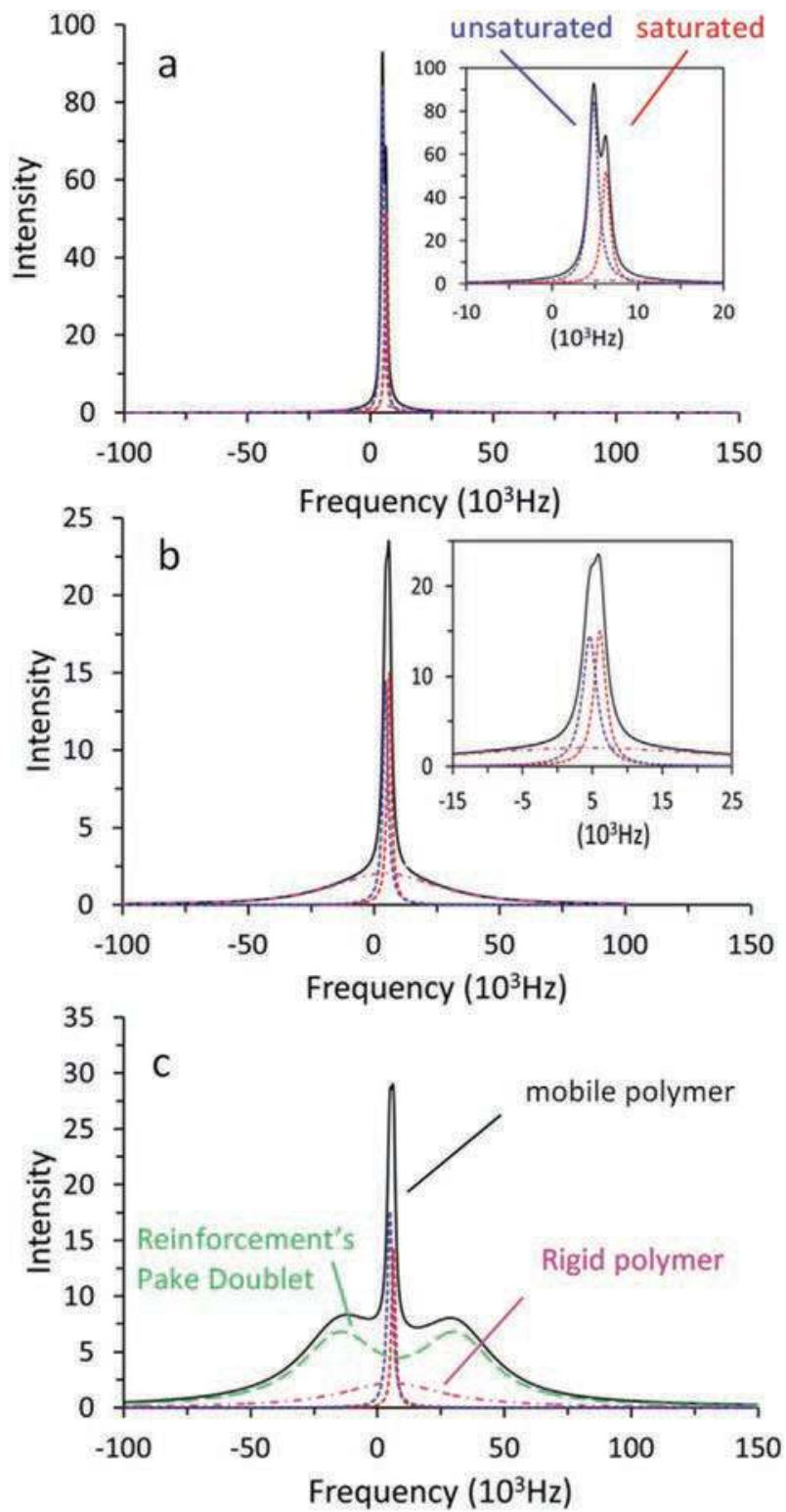

Figure 3. 'H NMR spectra obtained from a single pulse experiment on a) neat polymer with a recycle delay $\mathrm{RD}=5 \mathrm{~s}$, b) the composite with $\mathrm{RD}$ $=5 \mathrm{~s}$, and $\mathrm{c}$ ) the composite with $\mathrm{RD}=4800 \mathrm{~s}$. The non-continuous lines are theoretical ones that were deduced from a deconvolution procedure: the red and blue dotted lines are assigned to the mobile polymer fraction from saturated and unsaturated carbons; the pink dashed-dotted component is assigned to rigid polymer fraction (which is more intense in the composite); the green dashed line is the signal of the reinforcement, only visible with a long recycle delay in (c).

of the broader one. In the spectrum of the reinforcement, the broadened Pake doublet can be approximated by two individual Lorentzian lines. In the spectrum of the composite recorded with a short delay, a possible contribution of this Pake doublet can be seen, but its contribution is too weak to be clearly distinguished from the spectral deconvolution. The spectrum is thus deconvoluted with three lines, and the contribution of the reinforcement is estimated from pure reinforcement spectrum recorded under the same experimental conditions. We found that $4 \%$ of the total spectral integration comes from residual reinforcement. This contribution was removed from the broad line integration determined by the optimization procedure. Finally, integrations were corrected to deal with relaxation occurring during the dead time of $6.5 \mu \mathrm{s}$ before acquisition.

The $T_{2}$-relaxation measured using an independent Hahn echo experiment was fitted with four exponential components: a long $T_{2}$ (see Table 1 for numerical values) attributed for both sharp lines, two shorter $\mathrm{T}_{2}$ attributed to the broad line on the bottom, and a small proportion of a very long fourth $T_{2}(>5 \mathrm{~ms})$ probably due to some impurities not visible in the spectrum.

The results gathered in Table 1 show that the polymer contains $19 \%$ of rigid domains, but this proportion increases up to $59 \%$ in the composite. At the same time, an overall broadening of the spectrum is observed. The results on the pure reinforcement are not presented in this paper, as they do not contribute to the overall results, the reinforcement being suppressed during the diffusion experiment in the composite.

$T_{2}$ was used to determine diffusion coefficients in both phases according to Equation (5). The broad line consists of two unresolved contributions: from the polymer immobilized in the interphase close to the reinforcement, and from the small domains present in the polymer. The $80 \mu \mathrm{s}-T_{2}$ is attributed to the small domains and yields the coefficient of $0.22 \mathrm{~nm}^{2} \mathrm{~ms}^{-1}$. The smallest $T_{2}$ is attributed to the rigidified interphase and yields the diffusion coefficient of $0.53 \mathrm{~nm}^{2} \mathrm{~ms}^{-1}$.

\subsection{NMR Spin Diffusion Measurements}

Diffusion curves representing the evolution of $I_{\text {corr }}$ versus $t_{\mathrm{m}}{ }^{1 / 2}$ are displayed in Figure 4 for the composite and neat polymer. An evolution curve $I(t)_{\tau=50 \mu \mathrm{s}}$ before correction is given for comparison. One can observe the two-stage pattern of the composite's signal: a fast initial decay followed by a slower decay for longer mixing times. Different tests were carried out to ensure that the observed behavior did not result from experimental drift or artefacts. It was confirmed by using different sequences including the basic GS experiment with phase cycling, and the

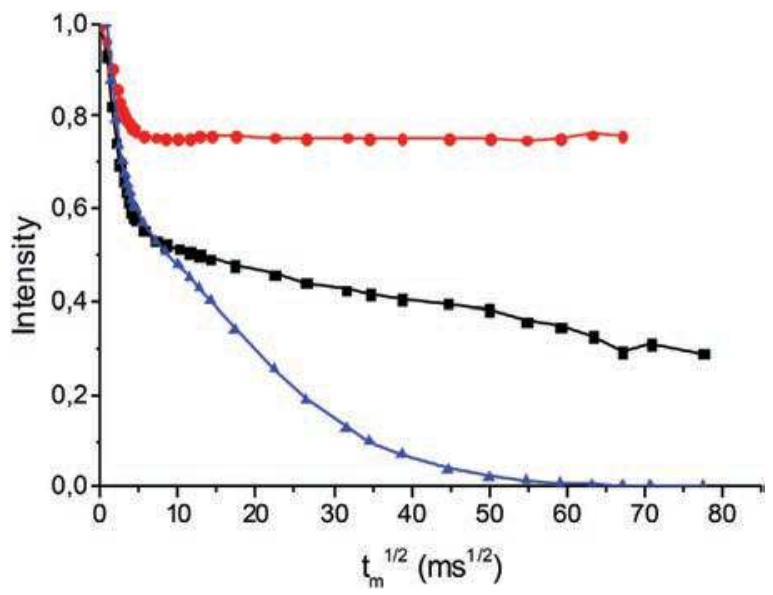

Figure 4. Corrected diffusion curves obtained with the sequence presented in Figure 1. Dots: pure polymer, squares: composite. The third curve (triangles) represents the diffusion curve before correction. 


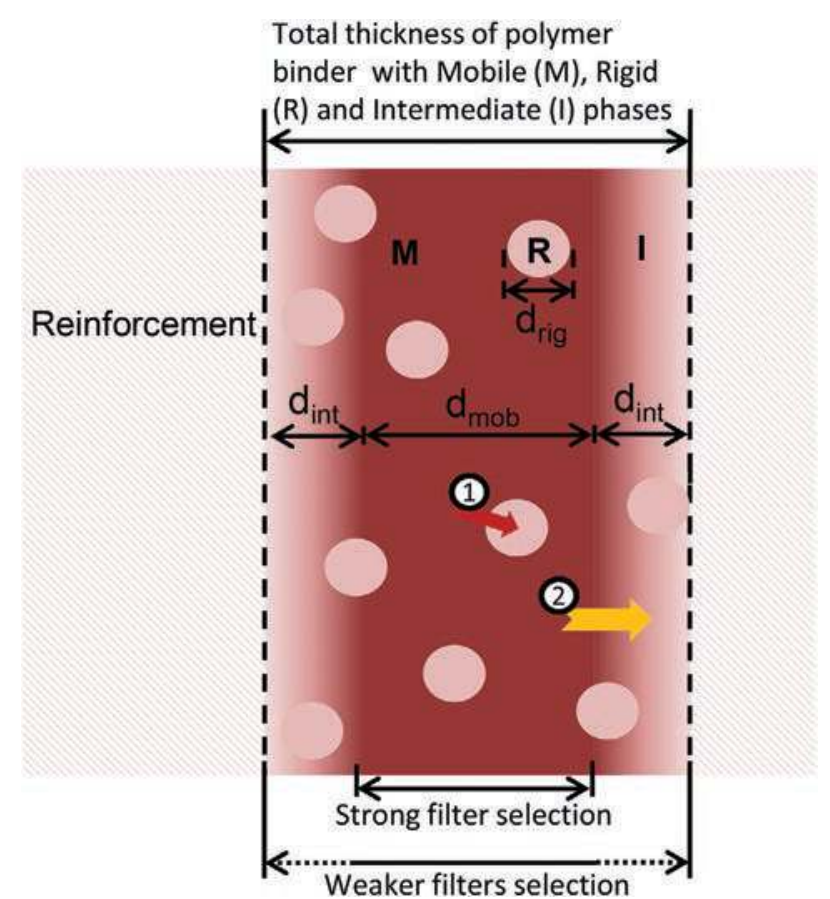

Figure 5. Suggested model for polymer binder between two reinforcement particles. The darker the material, the more mobile. $\mathrm{R}$ magnetization is cancelled with a short $\tau_{1}$; I magnetization is progressively cancelled with increasing $\tau_{1}$. Also shown is a fast diffusion from the source $M$ to the sink R (1), and a slower diffusion, considered as the diffusion of bulk "free" (M+R) polymer toward bulk rigidified polymer (2).

dipolar filter ${ }^{[27]}$ (not shown). The graph shows that a similar fast initial decay is observed in the polymer and in the composition even without $T_{1}$-correction. This correction is however required to reveal the slower decay of the composite, in opposition to the plateau final value observed for the neat polymer. A limit is reached at $t_{\mathrm{m}} \approx 3.5 \mathrm{~s}$, after which the noise increases dramatically. This is a consequence of the relaxation attenuation that affects both the numerator and denominator in Equation (1).

To the best of our knowledge, a similar two-stage evolution has only been reported once, for core-shell systems. ${ }^{[15]}$ The interpretation given relies on the coexistence of a two scale structuring. The fast decay is attributed to small rigid domains within the interphase, close to the core-shell interface, and the slower decay to the core-shell macro-structure itself. This interpretation is transposed here assuming that the polymer in the composite contains: i) small isolated rigid polymer domains as observed in the neat polymer; ii) an interphase of finite length made of rigidified polymer close to the reinforcement. Both play the role of sink phase for magnetization (Figure 5); hence following the magnetization of polymer chains, one should observe at the same time a fast local diffusion toward the small rigid domains, and a slower transfer of magnetization towards the rigidified interphase. Both processes are simultaneous, but the first one vanishes around $t_{\mathrm{m}} \approx 50 \mathrm{~ms}$ while the second one goes on for a few seconds. Thanks to this difference, and to go further in the analysis, we assume that the two diffusion processes are independent. The two-stage diffusion curves observed for each $\tau_{1}$ value were decomposed into separate fast and slow decays, and renormalized (see Figure 7a).

\subsection{Evaluation of Filter Strength and Selected Mobile Fraction}

The delays used for the selection period act as filters allowing the selection of more or less mobile fractions (Figure 5). With a weak filter, that is, with a short $\tau_{1}$, only the magnetization of the most rigid protons is cancelled. Hence, the maintained magnetization comes from a large region of the material. Concerning the interphase and assuming that $T_{2}$ relaxation times increase with the distance from the reinforcement, the region of cancelled magnetization grows with increasing $\tau_{1}$ delays, and the size of the selected regions decreases. The point where a constant $T_{2}$ is reached, far enough from the reinforcement, is defined as the edge of the interphase. The size distribution of domains is another factor that contributes to the observed $\tau_{1}$ dependency, and this effect probably prevails for small isolated domains related to the fast initial decay.

Experimentally, the selected fraction of polymer represents the protons from the mobile phase that have retained their magnetization after equilibrium is reached. The evolution curves obtained in neat polymer and composite are displayed in Figures 6 and 7. A similar initial decrease is observed, followed by a plateau value reached at $\tau_{1} \approx 50 \mu$ s (Figures 6a and $7 \mathrm{~b}$ ). Dealing with the slow diffusion in the composite, the selected
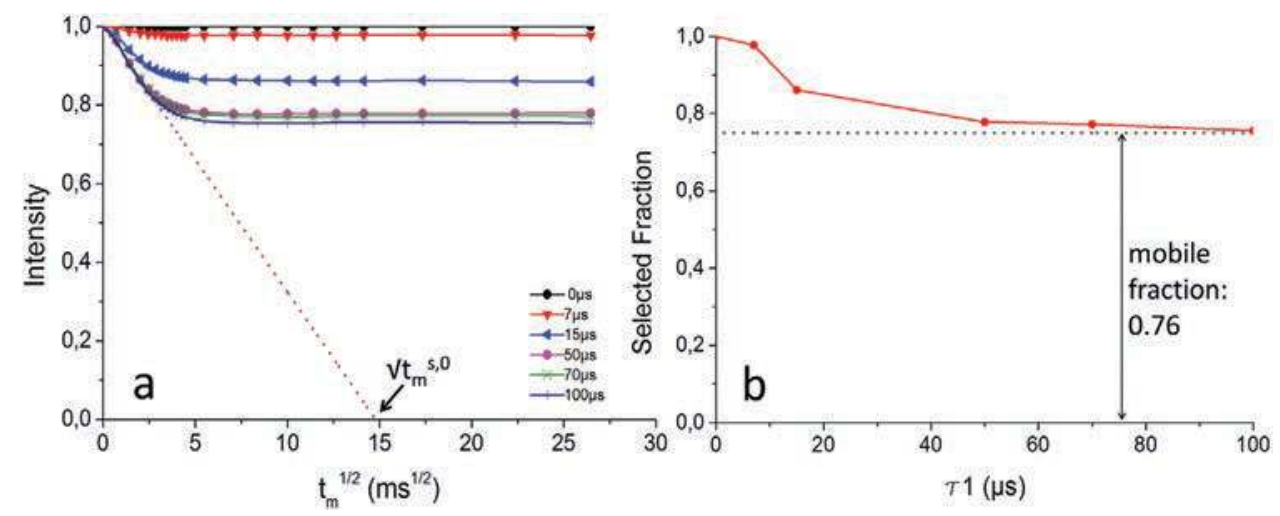

Figure 6. a) Diffusion curves of the polymer at different filter strengths $\left(\tau_{1}\right)$, with determination of the characteristic time for a strong filter. b) Selected mobile fraction versus $\tau_{1}$ in the neat polymer with a final value of 0.76 . 

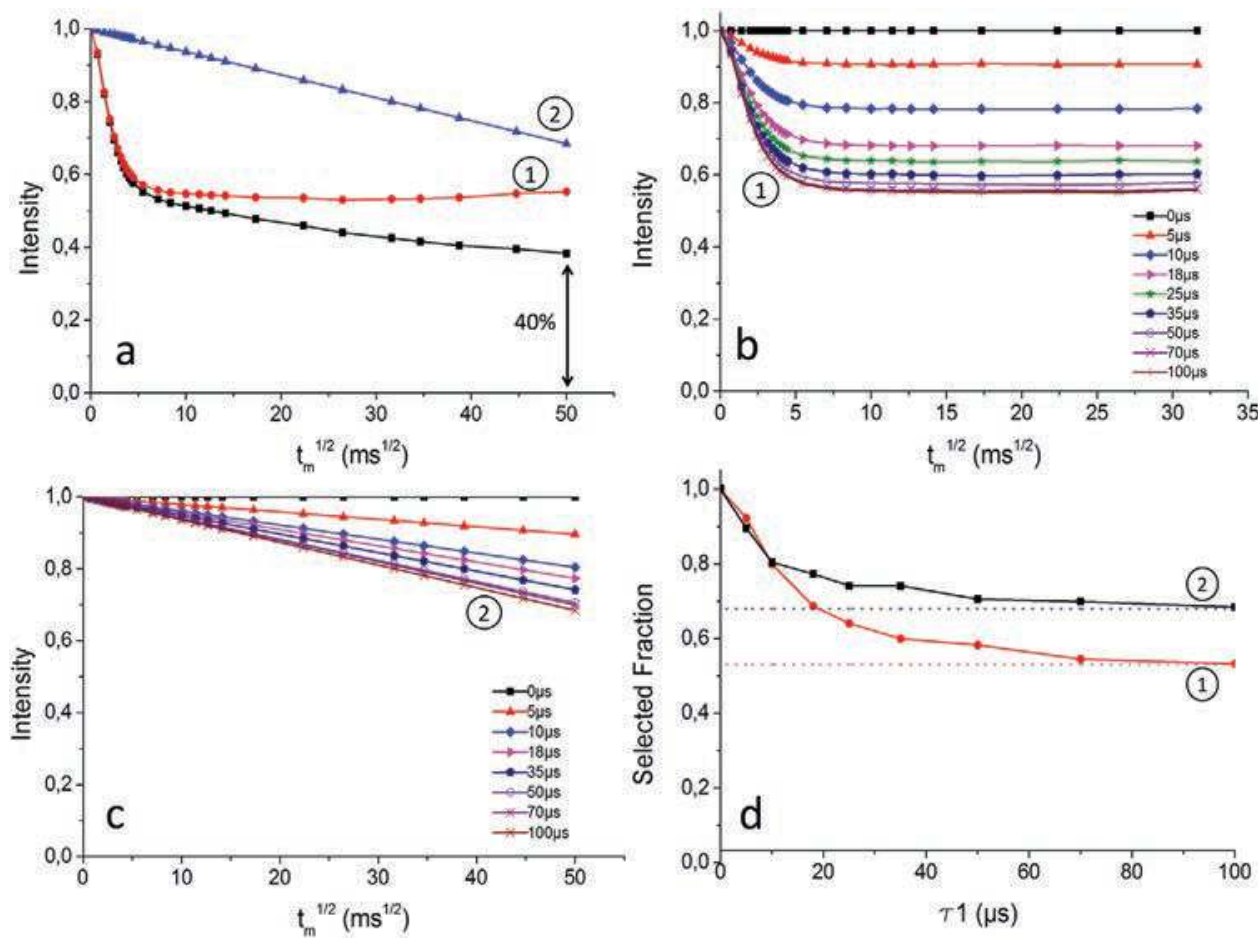

Figure 7. a) Total diffusion curve of the composition (squares) decomposed into two distinct regimes: a fast diffusion toward small domains (1) and a renormalized slower and longer diffusion toward the interphase (2). Fast (b) and slow (c) diffusion curves at different filter strengths. d) Selected mobile fractions for the two decays: respective final values of 0.53 (diffusion 1) and 0.68 (diffusion 2).

mobile fraction versus $\tau_{1}$ curve is generated using the intensity at $t_{\mathrm{m}}=2.5 \mathrm{~s}$, as equilibrium is not reached, even if this value gives an overestimation of the selected fraction.

The delay $\tau_{2}$ before acquisition has to be long enough to cancel the residual contribution from the reinforcement, and short enough not to add relaxation effects to the mobile component signal. It is optimized in a Hahn echo experiment and then set for all diffusion measurements at $50 \mu \mathrm{s}$.

\section{Discussion}

\subsection{Pure Polymer}

The diffusion curves of the polymer displayed in Figure 6 show the presence of rigid domains that represent about $25 \%$ of the polymer matrix, which is consistent with the quantitative analysis of the $1 \mathrm{D}$ spectrum. This is a common situation in polymers that contain separate phases, segregation of chemical species (for instance, aromatic and aliphatic species), entanglements, and crosslinks. For comparison purposes, the method was tested on a room temperature vulcanization silicone sample with simple chemical structure and low glass transition temperature. Such a polymer was expected not to contain any microstructure or gradient of molecular mobility, thus no diffusion was expected, and indeed no diffusion was observed regardless of the $\tau_{1}$ value. Information on domain size was determined using Equation (3) assuming the rigid domain is the dispersed component and diffusion coefficients from Table 1 . The proton densities are supposed distinct here: to a first approximation, the rigid domain is assigned to EPON and the mobile component to NBR chemical components.

Dealing with isolated rigid clusters related to a fast diffusion, an isotropic spheroidal geometry with $\varepsilon=3$ is assumed, leading to an average domain size $d_{\text {rig }}=6 \mathrm{~nm}$ (Figure 5). These spheroidal domains represent about $30 \%$ of the volume, considering the respective proton densities.

\subsection{Composite}

Figure 7 a displays the diffusion curve recorded at long $\tau_{1}$ on the composite material. The observation of a linear slow decay for long mixing times is the main difference from the neat polymer. The end value close to $40 \%$ matches the fraction deduced from the deconvolution of the 1D spectrum (see Table 1), that is, the domains involved in the diffusion process correspond to the narrow and broad lines in the ${ }^{1} \mathrm{H}$ spectrum.

After decomposition of the diffusion curve, the fast component shows almost the same evolution as in neat polymer but with a lower selected mobile fraction (Figure $7 \mathrm{~b}$ ). The domain size $d_{\text {rig }}$ is calculated from the same proton densities as for the polymer. It is determined to be $8 \mathrm{~nm}$, a value similar to that found for neat polymer, yet a little higher. These results express an overall stiffening of the binder within the composite. Considering that the first decay reaches equilibrium within $50 \mathrm{~ms}$, and turning now to the longer second decay, it turns out that this decay characterizes the diffusion that takes place between the bulk "free" binder and the bulk rigidified binder at the interface. Indeed, when the first diffusion between the mobile 
polymer $\mathrm{M}$ and the small rigid domains $\mathrm{R}$ contained within $M$ reaches equilibrium, only a small part of the second diffusion has occurred (Figure 7c). It is thus consistent to consider that the second diffusion happens from the bulk $(M+R)$ free binder toward the bulk rigidified polymer I that includes both initially mobile and rigid local domains. This means that the proton densities are equivalent in both phases and correspond to the average of the proton densities of individual components. Figure $7 \mathrm{~d}$ shows that the selected mobile fraction for the second decay is about $68 \%$, meaning that $32 \%$ of the protons are rigidified in the region close to the reinforcement. These are the volume fractions used in the calculations, even if these values are respectively over- and under-estimated since equilibrium is not reached in the considered mixing time. A dimensionality $\varepsilon=1$ is chosen considering the diffusion from a lamella of free binder towards a lamella of rigidified binder in contact with the reinforcement. Equation (3) gives a domain thickness $d_{\text {int }}=30 \mathrm{~nm}$, with the interphase as the rigid dispersed component; this result is a low limit of $d_{\text {int }}$ since $\phi_{\text {int }} / \phi_{\text {mob }}$ is underestimated. These $30 \mathrm{~nm}$ represent the thickness of one interphase, and now there must be an interphase on each side of the binder lamella where the binder is under the influence of the reinforcement. This means that the $32 \%$ of rigidified protons represent a total thickness of $2 \times 30 \mathrm{~nm}$, which enables us to estimate the proportional size of the free binder's domain at $128 \mathrm{~nm}$. Finally, the total thickness of the binder is estimated to $2 \times d_{\text {int }}+d_{\text {mob }}=188 \mathrm{~nm}$ (see Figure 5). Obviously, SEM observations showed that the polymer distribution is not homogeneous throughout the sample. This result has thus to be considered as a mean value resulting from the weighted average of individual slopes related to the different domain sizes encountered in the sample: from binder heaps of a few micrometers long and wide that give almost no diffusion (no slope) to thin lamellae not visible in SEM pictures, that give a rapid diffusion (steep slopes).

\section{Conclusion}

This work proved the complementarity between SEM observations and NMR spin diffusion experiments to characterize the microstructure of a composite material, and more precisely the structuring of the few percent of its polymer binder. SEM observations revealed the heterogeneity of the material, and showed the distribution of the binder in clusters a few micrometres square, mainly between melamine particles. Because of the characteristics of the studied material, SEM resolution being limited, NMR spin-diffusion experiments were run to go further. The two-decay spin diffusion curves indicated the existence of two distinct types of domains. Small rigid domains were represented by the first fast decay, while the second slow decay revealed the presence of large rigid domains that did not exist in the pure binder. These were interpreted as an interphase region made of polymer with reduced mobility. Assuming a lamellar morphology, the size of the interphase containing a mobility gradient was estimated at $30 \mathrm{~nm}$, and the bulk mobile domain at $128 \mathrm{~nm}$, which lead to an average thickness of $188 \mathrm{~nm}$ on the whole sample. Such structural information is essential for a better understanding of microstructure/property relationships and will be used to improve the accuracy of numerical models developed to predict performances. Work is currently ongoing in our laboratory to apply this methodology to different composite materials and to evaluate the effect of mechanical or thermal treatment on the microstructure. From a wider point of view, as polymer-based composites are found in different sectors, this approach might be used for a wide variety of studies dealing with their properties, making process, or mechanical behavior.

\section{Experimental Section}

The studied compound is a home-made compacted composite material containing a mixture of melamine $(65 \%)$ and barium sulfate $(30 \%)$ as reinforcement, and about $5 \%$ of a polymer binder. The crystalline particles of reinforcement are a few dozen micrometers in size. The binder is an elastomer principally made of NBR (butadiene/acrylonitrile rubber) and $\approx 20 \%$ of EPON (multifunctional aromatic epoxy resin). The glass transition temperature measured by differential scanning calorimetry (DSC) is $T_{\mathrm{g}}=233 \mathrm{~K}$. Cylindrical samples of $\phi 10 \mathrm{~h} 4$ and $\phi 4 \mathrm{~h} 25$ $\mathrm{mm}$ were prepared for SEM and NMR measurements, respectively. For SEM observations, samples were further polished with an argon-gun Gatan PECS II ionic polisher.

SEM data were acquired using a field emission gun FEI Versa 3D SEM, operating at $10 \mathrm{kV}$. A circular backscattered electron detector was used in order to enhance the chemical contrast. To preserve this contrast, the samples were not metallized; this leads to an accumulation of electric charge common in organic materials. The problem is overcome using low vacuum conditions with a reduced pressure of $0.7 \mathrm{mbar}$ in the chamber, a voltage of $10 \mathrm{kV}$, and a probe current of $5.7 \mathrm{nA}$. EDS cartographies were also run on the sample, to supplement SEM information. They were acquired using an Oxford XMAX50 system with the same parameters as for SEM images, with an exposure of $1 \mathrm{~ms}$ per pixel. The area investigated with both techniques measured $211 \times 145 \mu \mathrm{m}^{2}$ for a resolution of $1024 \times 704$ pixels.

NMR measurements were carried out at room temperature (294 K) on a 9.4T Bruker wide bore spectrometer (proton resonance frequency of $400.13 \mathrm{MHz}$ ) equipped with a ${ }^{1} \mathrm{H}$ selective $5 \mathrm{~mm}$ high-power probe. The $90^{\circ}$ pulse produced by the solenoid coil was $1.5 \mu \mathrm{s}$. The GS experiment was repeated for each of the 32 diffusion times $t_{\mathrm{m}}$ chosen in the interval $(0.5-6000 \mathrm{~ms})$. Individual spectra were acquired with 8 scans with a recycle delay $R D=5 \mathrm{~s}$. The $2 \mathrm{D}$ data treatment consists of an exponential multiplication (line broadening $L B=500 \mathrm{~Hz}$ ), a Fourier transform in the first dimension, and a phase correction. The diffusion decay curves were built with the area of the entire line, to get quantitative results. 1D spectra were also recorded using the same recycle time. This methodology was applied to the two pure components separately, and then to the composite material. An important characteristic of the composites is the strong difference of longitudinal relaxation times $T_{1}$ between the reinforcement and the polymer. As a consequence, with a recycle delay of $5 \mathrm{~s}$ between the scans, the reinforcement magnetization was saturated and did not contribute to the spectrum. A signal residue could be observed at long mixing times, but is eliminated using an appropriate dead time. A one-scan spectrum was also acquired on the reinforcement and on the composite after a delay $\mathrm{RD}=4800 \mathrm{~s}$, to obtain the spectral response of the reinforcement, the $T_{1}$ of which was estimated to be roughly $800 \mathrm{~s}$. This $T_{1}$ value was independently evaluated on neat reinforcement using the standard inversion-recovery sequence with a reduced recycle delay of $60 \mathrm{~s}$. This value was also consistent with a series of single scan standard spectra recorded after different recycle delays. The spectra were deconvoluted with the software DMFit to extract spectral characteristics.

\section{Acknowledgements}

The funding for this work was provided by the CEA. 


\section{Conflict of Interest}

The authors declare no conflict of interest.

\section{Keywords}

composites, interphase, microstructure, nuclear magnetic resonance, polymer, scanning electron microscopy, spin diffusion

[1] M. Goldman, L. Shen, Phys. Rev. 1966, 144, 321.

[2] R. A. Assink, Macromolecules 1978, 11, 1233.

[3] W. G Hu, K. Schmidt-Rohr, Polymer 2000, 41, 2979.

[4] L. Zhang, H. Tang, G. Hou, Y. Shen, F. Deng, Polymer 2007, 48, 2928.

[5] C. Hedesiu, D. E Demco, R. Kleppinger, A. A. Buda, B. Blümich, K. Remerie, V. M. Litvinov, Polymer 2007, 48, 763.

[6] P. Sun, Q. Dang, B. Li, T. Chen, Y. Wang, H. Lin, Q. Jin, D. Ding, Macromolecules 2005, 38, 5654.

[7] A. Kretschmer, R. Drake, M. Neidhoefer, M. Wilhelm, Solid State Nucl. Magn. Reson. 2002, 22, 204.

[8] P. Bergquist, J.-F. Shi, J. Zhao, A. A. Jones, P. T. Inglefield, R. P. Kambour, Macromolecules 1998, 31, 3632.

[9] F. Wang, R. Zhang, Q. Wu, T. Chen, P. Sun, A. C. Shi, ACS Appl. Mater. Interfaces 2014, 6, 21397.

[10] Q. Zou, L. Zhang, S. Li, X. Gao, F. Deng, J. Colloid Interface Sci. 2011, 355, 334.

[11] J. Brus, J. Dybal, P. Sysel, R. Hobzovà, Macromolecules 2002, 35, 1253.

[12] R. C. Nieuwendaal, Solid State Nucl. Magn. Reson. 2016, 76-77, 29.

[13] F. Mellinger, M. Wilhelm, H. W. Spiess, Macromolecules 1999, 32, 4686.

[14] W. Z. Cai, K. Schmidt-Rohr, N. Egger, B. Gerharz, H. W. Spiess,
Polymer 1993, 34, 267.

[15] K. Landfester, H. W. Spiess, Acta Polym. 1998, 49, 451.

[16] S. M. De Paul, J. Zwanziger, R. Ulrich, U. Wiesner, H. W. Spiess, J. Am. Chem. Soc. 1999, 121, 5727.

[17] D. E. Demco, A. Johansson, J. Tegenfeldt, Solid State Nucl. Magn. Reson. 1995, 4, 13.

[18] M. A. Voda, D. E. Demco, A. Voda, T. Schauber, M. Adler, T. Dabisch, A. Adams, M. Baias, B. Blümich, Macromolecules 2006, 39, 4802.

[19] A. Buda, D. E. Demco, M. Bertmer, B. Blümich, J. Phys. Chem. B 2003, 107, 5357.

[20] K. Schäler, M. Roos, P. Micke, Y. Golitsyn, A. Seidlitz, T. ThurnAlbrecht, H. Schneider, G. Hempel, K. Saalwächter, Solid State Nucl. Magn. Reson. 2015, 72, 50.

[21] M. Roos, K. Schäler, A. Seidlitz, T. Thurn-Albrecht, K. Saalwächter, Colloid Polym. Sci. 2014, 292, 1825.

[22] K. Saalwächter, Y. Thomann, A. Hasenhindl, Macromolecules 2008, 41, 9187.

[23] P. Caravatti, P. Neuenschwander, R. Ernst, Macromolecules 1985, 18, 119.

[24] K. Schmidt-Rohr, J. Clauss, H. W. Spiess, Macromolecules 1992, 25, 3273.

[25] S. Friebel, R. K. Harris, A. M. Kenwright, Magn. Reson. Chem. 1997, $35,290$.

[26] J. Clauss, K. Schmidt-Rohr, H. W. Spiess, Acta Polym. 1993, 44, 1.

[27] N. Egger, K. Schmidt-Rohr, B. Blümich, W. D. Domke, B. Stapp, J. Appl. Polym. Sci. 1992, 44, 289.

[28] M. Mauri, Y. Thomann, H. Schneider, K. Saalwächter, Solid State Nucl. Magn. Reson. 2008, 34, 125. 